\section{Psychometrische Fragebögen zum Befinden}

Tina Steitz ${ }^{1}$ und Hans-Günter Wee $\beta^{2}$

${ }^{1}$ Abteilung Strafvollzug, Ministerium der Justiz RheinlandPfalz, Mainz, Deutschland

${ }^{2}$ Interdisziplinäres Schlafzentrum Pfalzklinikum, Klingenmünster, Deutschland

\section{Synonyme}

Psychometrische Untersuchungsverfahren

\section{Englischer Begriff}

psychometric questionnaires

\section{Definition}

Psychometrische Fragebögen zum Befinden dienen der Erfassung des emotionalen Zustands des Probanden. Es wird zwischen der Erfassung des allgemeinen emotionalen Befindens und der Erfassung bestimmter Aspekte des Befindens unterschieden. Dabei können beispielsweise depressives Erleben oder das Vorliegen einer erhöhten Ängstlichkeit als spezifische Aspekte des emotionalen Befindens verstanden werden. Weiterhin kann zwischen State- und Trait-Messungen unterschieden werden. Bei der Trait-Messung wird das emotionale Befinden als ein über die Zeit andauerndes Merkmal über einen längeren Zeitraum erfasst, wohingegen die StateMessung sich auf rasche Schwankungen des jeweiligen aktuellen Befindens bezieht.

Folgende Verfahren erfassen unterschiedliche Aspekte des emotionalen Befindens und sind in separaten Essays dargestellt:

- >Befindlichkeitsskala“;

- „Basler Befindlichkeitsskala“;

- $>$ „Profile of Mood States“;

- $>$,Eigenschaftswörterliste“;

- $>$,State-Trait-Angstinventar“.

Siehe dazu auch $>$ „Psychodiagnostische Fragebögen“; >,Leistungs-, Schläfrigkeits- und Vigilanzmessung“; > „Leistungstests und Fahrtauglichkeitsprüfung““.

\section{Literatur}

Bullinger M, Morfeld M, Hoppe-Tarnowski D (2003) POMS. Profile of Mood States. In: Schumacher J, Klaiberg A, Braehler E (Hrsg) Diagnostische Verfahren zu Lebensqualität und Wohlbefinden. Hogrefe, Göttingen, S 262-264

Hobi V (1985) Basler Befindlichkeitsskala. Beltz, Weinheim

Janke W, Debus G (1978) Die Eigenschaftswörterliste EWL. Hogrefe, Göttingen

McNair DM, Lorr M, Droppelman LF (1971) Manual for the profile of mood states. CA Educational and Industrial Testing Service, San Diego

Zerssen D von, Koeller DM (1976) Die Befindlichkeits-Skala. Parallelformen Bf-S und Bf-SI aus: Klinische Selbstbeurteilungs-Skalen (Ksb-S) aus dem Münchener Psychiatrischen Informations-System (PSYCHIS München) Beltz, Weinheim 